\title{
Particles on Demand for Kinetic Theory
}

\author{
B. Dorschner, F. Bösch, and I. V. Karlin ${ }^{\dagger}$ \\ Department of Mechanical and Process Engineering, ETH Zurich, 8092 Zurich, Switzerland
}

(Received 12 June 2018; revised manuscript received 3 August 2018; published 25 September 2018)

\begin{abstract}
A novel formulation of fluid dynamics as a kinetic theory with tailored, on-demand constructed particles removes restrictions on flow speed and temperature as compared to its predecessors, the lattice Boltzmann methods and their modifications. In the new kinetic theory, discrete particles are determined by a rigorous limit process which avoids ad hoc assumptions about their velocities. Classical benchmarks for incompressible and compressible flows demonstrate that the proposed discrete-particles kinetic theory opens up an unprecedented wide domain of applications for computational fluid dynamics.
\end{abstract}

DOI: 10.1103/PhysRevLett.121.130602

The kinetic theory of Boltzmann and Maxwell, as the fundamental link between the particles' picture of flowing matter and a continuum projection thereof, has been a valuable source of ideas in fluid dynamics. This particularly concerns the lattice Boltzmann method (LBM) [1-4]. LBM is a recast of fluid mechanics into a kinetic theory for the populations of designer particles $f_{i}(\boldsymbol{x}, t)$, with simple rules of propagation on a space-filling lattice formed by discrete speeds $\mathcal{C}=\left\{\boldsymbol{c}_{i}, i=1, \ldots, Q\right\}$, in discrete-time $t$, and relaxation to a local equilibrium $f_{i}^{\text {eq }}(\boldsymbol{x}, t)$ at the nodes $\boldsymbol{x}$. LBM witnessed a rapid growth in applications such as multiphase flow [5-7], microfluidics [8,9], hemodynamics [10], or turbulence [11], to mention a few; for reviews, see Refs. [12-14].

However, a critical look at LBM reveals major limitations: all practical LBM models are severely restricted in flow speed and temperature range. While these restrictions may be traded for deeply subsonic, slow flows, even then insufficient isotropy and the lack of Galilean invariance impede simulations [15]. Moreover, the said limitations become eventually insurmountable for compressible flows [16-20]. It may be argued that LBM has reached its natural limits with the simulation of quasi-incompressible flows, and a different discrete kinetic theory is needed for important fields such as combustion and aerodynamics.

In this Letter, we demonstrate that eventually all physical limitations of the LBM are removed once the discrete kinetic theory is formulated using tailored rather than fixed particles' velocities. The new fully explicit realization is seamless; it requires no a priori information on the type of flow and sets a rigorous upper bound on the flow speed and temperature that can be achieved with a given set of discrete speeds.

We begin with a clarification: LBM interprets the discrete speeds $\boldsymbol{c}_{i}$ as particles' velocities, $\boldsymbol{v}_{i}^{L}=\boldsymbol{c}_{i}$. On the contrary, here we understand $\boldsymbol{c}_{i}$ as peculiar velocities [21], relative to a reference frame velocity $\boldsymbol{u}$ and a temperature $T$. Henceforth, particles' velocities are defined as

$$
v_{i}=\sqrt{\theta} c_{i}+u,
$$

where $\theta=T / T_{L}$ is the temperature reduced by the lattice temperature $T_{L}$, a constant which is known for any set of discrete speeds $\mathcal{C}$ [22]. According to (1), LBM amounts to setting a global reference frame "at rest," $\boldsymbol{u}=\mathbf{0}$, and choosing the fixed temperature $T=T_{L}$ for all particles. Here, we rather follow the interpretation (1) where the reference frame velocity and temperature are kept so far undetermined, and we are going to find optimal values for $\boldsymbol{u}$ and $T$ locally at every monitoring point, as presented in detail below.

By specifying the frame velocity and temperature in (1), one sets the reference frame (or gauge) $\lambda=\{\boldsymbol{u}, T\}$ for the discrete velocities. LBM corresponds to the standard gauge $\lambda_{L}=\left\{\boldsymbol{0}, T_{L}\right\}$. We denote $f^{\lambda}=\left(f_{1}^{\lambda}, \ldots, f_{Q}^{\lambda}\right)^{\dagger}$ as the vector of populations relative to the gauge $\lambda$. The transform of the populations to another gauge $\lambda^{\prime}=\left\{\boldsymbol{u}^{\prime}, T^{\prime}\right\}$ is facilitated by matching $Q$ linearly independent moments $(m, n$ are integers; $D=2$ to ease notation),

$$
M_{m n}^{\lambda}=\sum_{i=1}^{Q} f_{i}^{\lambda}\left(\sqrt{\theta} c_{i x}+u_{x}\right)^{m}\left(\sqrt{\theta} c_{i y}+u_{y}\right)^{n} .
$$

Let us use a shorthand notation for a linear map of populations into moments $M^{\lambda}$ (2), $M^{\lambda}=\mathcal{M}_{\lambda} f^{\lambda}$, where $\mathcal{M}_{\lambda}$ is the $Q \times Q$ matrix of the linear map. The matching condition for the moments in both gauges $\lambda$ and $\lambda^{\prime}$ reads

$$
M^{\lambda}=M^{\lambda^{\prime}} .
$$

In other words, the moments of the populations are requested to be independent of the choice of a gauge, analogously to the independence of continuous-velocity moments (integral quantities) of the integration variable. Moments matching condition (3) imply that populations are transformed from one gauge to another with the transfer matrix $\mathcal{G}_{\lambda}^{\lambda^{\prime}}$, 


$$
f^{\lambda^{\prime}}=\mathcal{G}_{\lambda}^{\lambda^{\prime}} f^{\lambda}=\mathcal{M}_{\lambda^{\prime}}^{-1} \mathcal{M}_{\lambda} f^{\lambda} .
$$

Finally, we introduce a reconstruction formula for populations at any point $\boldsymbol{x}$ at time $t$ :

$$
\tilde{f}^{\lambda}(\boldsymbol{x}, t)=\sum_{s=1}^{k} a_{s}\left(\boldsymbol{x}-\boldsymbol{x}_{s}\right) \mathcal{G}_{\lambda_{s}}^{\lambda} f^{\lambda_{s}}\left(\boldsymbol{x}_{s}, t\right)
$$

where summation is carried over collocation points $\boldsymbol{x}_{s} ; \lambda_{s}$, $s=1, \ldots, k$ corresponds to local gauges at the collocation points at time $t, \lambda_{s}=\left\{\boldsymbol{u}\left(\boldsymbol{x}_{s}, t\right), T\left(\boldsymbol{x}_{s}, t\right)\right\}$, and $a_{s}$ are interpolation functions (standard Lagrange polynomials were used; $k$ determines the order, $k=3$ below). Note that the reconstruction formula (5) enforces populations at collocation points to be treated in a specified gauge $\lambda$ through the transform (4).

We now present the discrete kinetic theory in an optimal local gauge. Introducing the time step $\delta t$, evaluation of the populations at the monitoring point $\boldsymbol{x}$ at time $t$ involves the propagation and the collision steps, mediated by the gauge transform.

Propagation.-Semi-Lagrangian advection is performed first, using the reconstruction formula (5) at the departure point of characteristic lines, $\boldsymbol{x}-\boldsymbol{v}_{i}^{0} \delta t$,

$$
f_{i}^{\lambda_{0}}=\tilde{f}_{i}^{\lambda_{0}}\left(\boldsymbol{x}-\boldsymbol{v}_{i}^{0} \delta t, t-\delta t\right),
$$

where the characteristic directions $\boldsymbol{v}_{i}^{0}$ [or discrete velocities, cf. Eq. (1)] are set relative to a seed gauge $\lambda_{0}=\left\{\boldsymbol{u}_{0}, T_{0}\right\}$. For the latter, it is convenient to choose flow velocity and temperature at the monitoring point $\boldsymbol{x}$ at time $t-\delta t$ :

$$
\boldsymbol{u}_{0}=\boldsymbol{u}(\boldsymbol{x}, t-\delta t), \quad T_{0}=T(\boldsymbol{x}, t-\delta t),
$$

yielding

$$
v_{i}^{0}=\sqrt{\theta_{0}} c_{i}+u_{0},
$$

with $\theta_{0}=T_{0} / T_{L}$. Since $\boldsymbol{u}_{0}$ and $T_{0}$ are known from the previous time step, the populations (6) are determined unambiguously in this predictor propagation step.

With the populations (6), the density, momentum, and temperature are evaluated at the monitoring point using discrete velocities (8):

$$
\begin{gathered}
\rho_{1}=\sum_{i=1}^{Q} f_{i}^{\lambda_{0}}, \\
\rho_{1} \boldsymbol{u}_{1}=\sum_{i=1}^{Q} \boldsymbol{v}_{i}^{0} f_{i}^{\lambda_{0}}, \\
D \rho_{1} T_{1}+\rho_{1}\left\|\boldsymbol{u}_{1}\right\|^{2}=\sum_{i=1}^{Q}\left\|\boldsymbol{v}_{i}^{0}\right\|^{2} f_{i}^{\lambda_{0}} .
\end{gathered}
$$

This defines the corrector gauge $\lambda_{1}=\left\{\boldsymbol{u}_{1}, T_{1}\right\}$ at the monitoring point, and advection (6) is executed anew with the updated velocities, $\boldsymbol{v}_{i}^{1}=\sqrt{\theta_{1}} \boldsymbol{c}_{i}+\boldsymbol{u}_{1}$, to get corrected postpropagation populations $f_{i}^{\lambda_{1}}$. The predictor-corrector process is iterated until convergence, with the limit values, $\rho(\boldsymbol{x}, t), \boldsymbol{u}(\boldsymbol{x}, t), T(\boldsymbol{x}, t), f_{i}^{\lambda(\boldsymbol{x}, t)}=\lim _{n \rightarrow \infty} \rho_{n}, \boldsymbol{u}_{n}, T_{n}, f_{i}^{\lambda_{n}}$, defining the density, the flow velocity, the temperature, and the precollision populations, respectively, at the monitoring point $\boldsymbol{x}$ at time $t$. Note that, by construction, the limit gauge $\lambda(\boldsymbol{x}, t)=\{\boldsymbol{u}(\boldsymbol{x}, t), T(\boldsymbol{x}, t)\}$ is the comoving reference frame in which the discrete particle's velocity (1) is defined by the values of the flow velocity and of the temperature at the monitoring point.

Collision.-In the comoving reference frame, the local equilibrium populations are defined by the density only,

$$
f_{i}^{\mathrm{eq}}=\rho W_{i},
$$

where the weights $W_{i}$ are known for any discrete speeds set $\mathcal{C}$ [22]. Hence, precollision populations are transformed to postcollision as

$$
f_{i}(\boldsymbol{x}, t)=f_{i}^{\lambda(\boldsymbol{x}, t)}+2 \beta\left[\rho(\boldsymbol{x}, t) W_{i}-f_{i}^{\lambda(\boldsymbol{x}, t)}\right],
$$

for the Bhatnagar-Gross-Krook (BGK) collision model. The relaxation parameter $\beta$ is related to the kinematic viscosity by $\nu=T(1 / 2 \beta-1 / 2) \delta t$. By fixing the temperature and canceling the energy corrections (11), one arrives at the isothermal version of the proposed kinetic theory. Comments are in order here: (i) In LBM, particles (represented by discrete velocities) are fixed once and for all with the identification $\boldsymbol{v}_{i}^{L}=\boldsymbol{c}_{i}$. Then the local equilibrium acquires noninvariant dependence on the flow velocity and temperature which leads to errors once $\boldsymbol{u} \neq \mathbf{0}$ and $T \neq T_{L}$. Accumulation of these errors is also the primary source of numerical instabilities when the plain BGK collision model is used in LBM. On the contrary, the new representation of kinetics creates "optimal particles" (or optimal discrete velocities), specific to each monitoring point at a given time (see propagation step) so that the equilibrium (12) "seen" by the populations becomes exact. Hence, this new representation is, in principle, restricted neither in the flow speed nor in the range of temperature variation. Error-free equilibrium can also result in unconditional numerical stability when using the BGK model. Below, we shall probe all this with benchmark simulations. (ii) If the standard gauge $\lambda_{L}$ is adopted, then the transfer matrix $\mathcal{G}$ is dropped in (5), and advection (6) becomes $f_{i}=\tilde{f}_{i}\left(\boldsymbol{x}-\boldsymbol{c}_{i} \delta t, t-\delta t\right)$. The latter, together with finite element reconstruction, was used in a recent semiLagrangian LBM (SLLBM) [23]. SLLBM is not restricted to space-filling lattices and was realized on body-fitted unstructured meshes [24], an obvious advantage if turbulent flow simulations are concerned. Previous studies 
[23-25] demonstrate that semi-Lagrangian reconstruction features low numerical dissipation; neither the gauge transform nor the BGK collision contribute to spurious dissipation.

The standard two-dimensional nine-speed set $D 2 Q 9$ was used in all simulations below and the BGK collision (13) was implemented for both isothermal and compressible flow. The transfer matrix was found in closed form and is presented in the Supplemental Material [26], which includes Refs. [27-29], together with the reconstruction formula realization and the hydrodynamic equations recovered by this model. The LBM time step $\delta t=1$ was used in all simulations.

First, we measured kinematic viscosity at isothermal conditions. The decay of plane shear wave with initial profile $u_{\xi}(\xi, \eta)=A \sin (2 \pi \xi / L), A=0.05$, in transverse direction and advection $u_{\eta}(\xi, \eta)=\mathrm{Ma}_{a} \sqrt{T}$ in the wavevector (longitudinal) direction was studied. The wave vector was rotated by $\pi / 4$ with respect to the standard Cartesian $x$ axis and periodic boundary conditions were applied in both longitudinal and transverse directions. This tilted-wave setup is standard to probe isotropy and Galilean invariance [30,31]: kinematic viscosity should not depend on the advection Mach number $\mathrm{Ma}_{a}$. An equidistant mesh with a resolution of $L=200 \sqrt{2}$ in the longitudinal direction was used. Kinematic viscosity was measured by a least square fit of the exponentially decaying function. In Fig. 1, the kinematic viscosity is shown for various temperatures, in a wide range of advection speeds. It is apparent that the results of the present formulation are in excellent agreement with theoretical prediction, for advection Mach numbers even as high as $\mathrm{Ma}_{a}=100$, and are independent of temperature. This is in sharp contrast to the standard lattice BGK (LBGK) [4] which shows a lack of Galilean invariance already at $\mathrm{Ma}_{a} \gtrsim 0.1$. While the latter

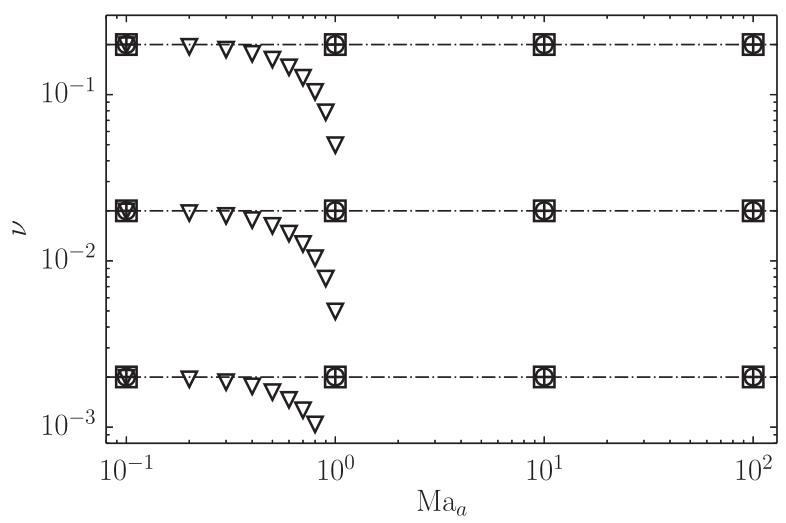

FIG. 1. Kinematic viscosity from decaying $\pi / 4$-tilted shear wave with $D 2 Q 9$, at various advection Mach numbers $\mathrm{Ma}_{a}=u / \sqrt{T}$. Lattice temperature $T_{L}=1 / 3$. Lines: imposed theoretical values $\nu=0.2, \nu=0.02, \nu=0.002$. Symbol: present method at fixed temperature $T_{L} / 2$ (cross), $T_{L}$ (circle), and $2 T_{L}$ (square). Triangle: LBGK [4] at $T_{L}$. failure of LBM has been long known [30,31], it is striking that a mere reformulation of the same kinetic model in the optimal gauge extends validity by at least 3 orders of magnitude in terms of flow speed. Note that, since the temperature can be set at a high value, and not only at $T=T_{L}$ as in the LBM, the quasi-incompressible flow simulations can be performed at realistic Mach numbers with the present method. This was used in the Green-Taylor vortex simulation at $\mathrm{Ma} \sim 10^{-3}$ which confirmed secondorder convergence, see Supplemental Material [26].

We now turn to the compressible flow while still using the nine-speed set $D 2 Q 9$. The difference with the above isothermal model is that now the energy conservation (11) is included in the predictor-corrector propagation step of the algorithm. The LBM counterpart is the thermal LBGK [32]. The first numerical experiment concerns measuring the speed of sound and comparing it to the theoretical prediction, $c_{s}=\sqrt{\gamma T}$, where the adiabatic exponent $\gamma=2$ for two-dimensional ideal gas. To that end, the speed of sound was measured by introducing a pressure disturbance $\Delta p=10^{-3}$ and tracking the resulting shock front. The results for a fluid at rest, and advected with $\mathrm{Ma}_{a}=10$ are presented in Fig. 2. It is apparent that the speed of sound measured in the simulation excellently agrees with theory for all temperatures in the range $T \in\left[10^{-4}, 10^{2}\right]$, irrespective of the advection speed. Figure 2 also shows that the thermal LBGK with nine speeds matches the correct speed of sound only at the lattice temperature $T=T_{L}$ [33]. Thus, the present method extends the physical relevance of thermal $D 2 Q 9$ LBGK by about six decades in terms of temperature range without changing the number of particles. In general, simulations of compressible flows with LBM require higher-order lattices, with a much larger number of discrete speeds [34-37]. We now compare the above nine-speed $D 2 Q 9$ model with the entropic LBM on a higher-order lattice with forty-nine speeds, D2Q49 [34]. The benchmark consists of the advection of a vortex by a

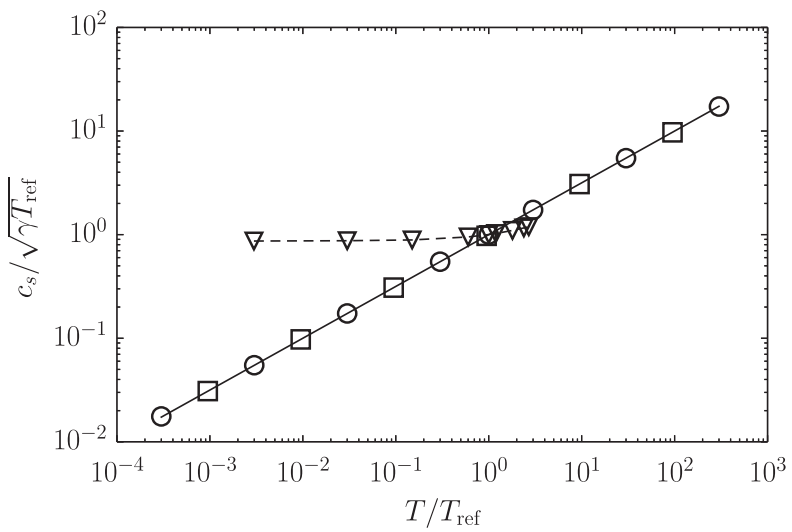

FIG. 2. Speed of sound with $D 2 Q 9$. Line: theory, $c_{s}=\sqrt{2 T}$. Circle: present method without advection. Square: present method with an advection Mach number $\mathrm{Ma}_{a}=10$. Triangle: thermal LBGK [33]. 


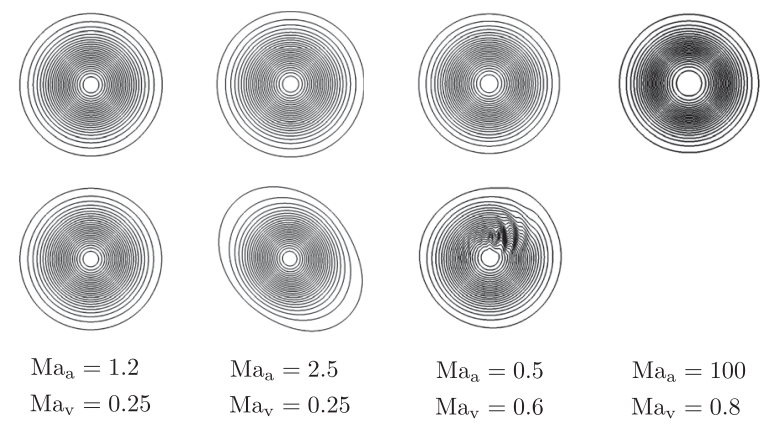

FIG. 3. Pressure contours of the vortex propagation for various advection and vortex Mach numbers. (Top row) present method, D2Q9; (Bottom row) entropic LBGK, D2Q49 [34].

uniform flow. The vortex with radius $R$ is propagated with advection Mach number $\mathrm{Ma}_{a}=U_{\infty} / \sqrt{2 T_{\infty}}$ while the vortex Mach number $\mathrm{Ma}_{v}$ defines the tangential velocity of the vortex $u_{\varphi}(r)=\mathrm{Ma}_{v} r \exp \left[\left(1-r^{2}\right) / 2\right]$, where $r=r^{\prime} / R$ is the reduced radius $[38,39]$. In Fig. 3, pressure contours are shown for the present $D 2 Q 9$ model (top row), together with those computed by the entropic LBM D2Q49 [34] (bottom row), for various combinations of $\mathrm{Ma}_{a}$ and $\mathrm{Ma}_{v}$. Note that LBM [34] is in a global gauge $\lambda=\left\{\boldsymbol{U}, T_{L}\right\}$, $\boldsymbol{U}=(1,0)$; this minimizes errors whenever $u_{x} \sim 1$. Clearly, with a global gauge conveniently chosen, unidirectional advection at small vortex Mach numbers can be accomplished with LBM (Fig. 3, first column). However, deviations of the local velocity and temperature away from the global gauge eventually lead to spurious deformation of the vortex (Fig. 3, second and third column). In contrast, the present method shows no deformation of the propagating vortex, even for large Mach numbers (Fig. 3, last column). This shows superiority of the present method over the higher-order LBM. Other pertinent aspects were studied using this benchmark. We observed that the predictorcorrector tailoring of the particles required about two to three iterations to convergence, with a maximum of five at a fraction of the grid points, when the gauge was initialized as in (7); see Supplemental Material [26]. The independence of the limit from the seed gauge was probed by choosing different values of $\boldsymbol{u}_{0}$ and $T_{0}$; e.g., $\boldsymbol{u}_{0}=\mathbf{0}, T_{0}=T_{L}$, or even "unnatural" $\boldsymbol{u}_{0}=-\boldsymbol{u}(\boldsymbol{x}, t-\delta t)$. We found that converged values are independent of the initialization which reveals that flow density, velocity, and temperature are indeed defined correctly. Thus, we can view the particles as an attractor of the predictor-corrector process. The basin of the attractor depends on the Mach number and narrows down at larger values; however, the seed gauge (7) was always included in the basin. This shows the robustness of the emerging kinetic picture.

The collision step retains locality and advanced collision models already elaborated in LBM are straightforward to apply in the present context. We conclude this Letter with a simulation of sound generation by a vortex passing through
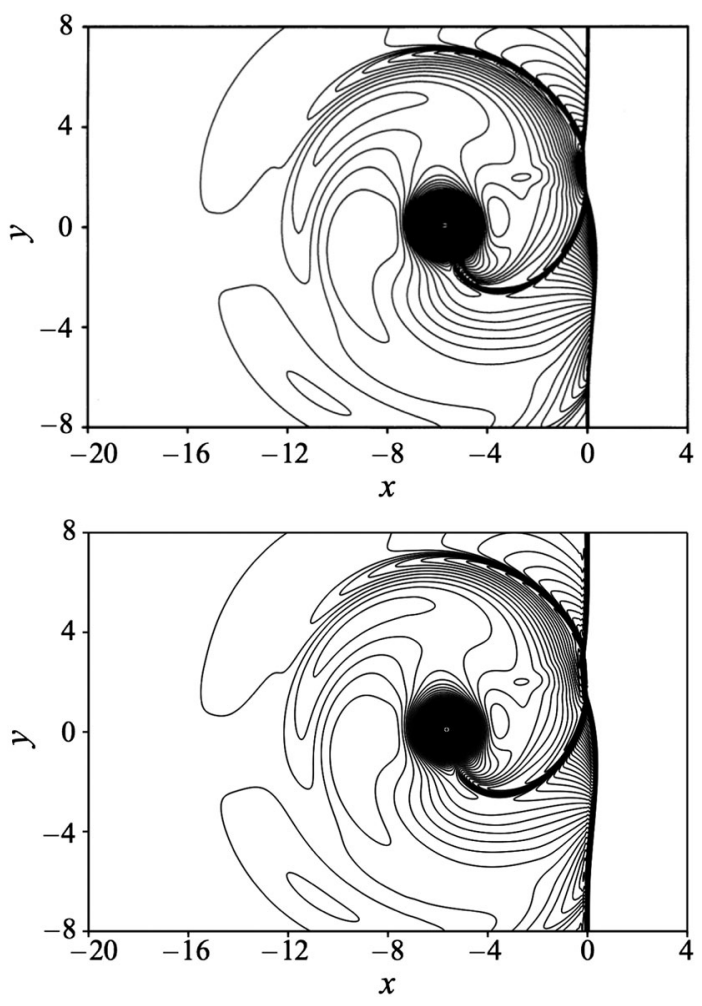

FIG. 4. Vortex-shock interaction. Snapshot of density contours for the case of vortex propagated with $\mathrm{Ma}_{a}=1.2, \mathrm{Ma}_{v}=0.5$, and $\operatorname{Re}=400$. Contour levels are from $\rho_{\min }=0.92$ to $\rho_{\max }=$ 1.55 with an increment of $\Delta \rho=0.0053$. (Top) DNS [39]; (Bottom) present.

a shock wave. To compare with the direct numerical simulation (DNS) of [39], we implemented the twopopulation (tunable adiabatic exponent) two-relaxation time (variable Prandtl number) collision model of Ref. [40] in the present setting using the $D 2 Q 25$ velocity set formed by roots of the fifth-order Hermite polynomial [32]. (The transfer matrix has a similar to $D 2 Q 9$ simple structure; details shall be reported in a follow-up paper.) In Fig. 4, density $\rho$ is compared with the DNS [39] at the reduced time $t=8$ (time in units of $R / a_{\infty}$, where $R=60$ is the radius of the vortex and $a_{\infty}=1$ is the speed of sound upstream of the shock). One can see the deformed shock front, the passing vortex, and reflected shocks developing, one of which remains connected to the vortex at this time. Excellent comparison between DNS and the present method is apparent; see also the Supplemental Material [26] for a further comparison of sound pressure.

Summarizing, the LBM is rigorously valid in the limit of vanishing flow velocity and at fixed lattice temperature. Practitioners of LBM circumvent these limitations by setting empirical bounds on the allowed variation of velocity and temperature (e.g., the flow velocity to stay below ten percent of the lattice speed of sound, $\|\boldsymbol{u}\| \lesssim$ $0.1 \sqrt{T_{L}}$, a common recommendation for incompressible flow simulations; see Fig. 1). However, such heuristic 
constraints cannot be universally maintained and quickly become meaningless, especially for compressible flows. We proposed a major revision of the kinetic theory for fluid dynamics by constructing "particles on demand" instead of a priori fixed. Its realization demonstrates that the range of accessible flow velocities and temperatures becomes restricted only by the choice of lattice speeds rather than by the closeness of flow parameters to $\boldsymbol{u}=0$ and $T=T_{L}$. The new discrete kinetic theory necessarily abandons the LBM lattice propagation since tailoring particles' velocities does not match to the links of a lattice. While the propagation step becomes computationally more intensive, the algorithm is still fully explicit, and the operation domain is incomparably larger. Finally, error-free collision features numerical stability even with the simplest BGK model. This all, as we believe, opens up a new perspective on complex flow simulations.

This work was supported by the SNF Grants No. P2EZP2_178436 (B.D.) and No. 200021-172640 (F.B.), and the Eidgenössische Technische Hochschule (ETH) research Grant No. ETH-13 17-1. Computational resources at the Swiss National Super Computing Center (CSCS) were provided under Grant No. s800.

*Present address: California Institute of Technology, Pasadena, California 91125, USA.

† Corresponding author.

karlin@lav.mavt.ethz.ch

[1] U. Frisch, B. Hasslacher, and Y. Pomeau, Phys. Rev. Lett. 56, 1505 (1986).

[2] G. R. McNamara and G. Zanetti, Phys. Rev. Lett. 61, 2332 (1988).

[3] F. J. Higuera, S. Succi, and R. Benzi, Europhys. Lett. 9, 345 (1989).

[4] Y.-H. Qian, D. d'Humières, and P. Lallemand, Europhys. Lett. 17, 479 (1992).

[5] L. Biferale, P. Perlekar, M. Sbragaglia, and F. Toschi, Phys. Rev. Lett. 108, 104502 (2012).

[6] M. Sbragaglia, R. Benzi, L. Biferale, S. Succi, and F. Toschi, Phys. Rev. Lett. 97, 204503 (2006).

[7] R. Benzi, S. Chibbaro, and S. Succi, Phys. Rev. Lett. 102, 026002 (2009).

[8] C. Kunert and J. Harting, Phys. Rev. Lett. 99, 176001 (2007).

[9] J. Hyväluoma and J. Harting, Phys. Rev. Lett. 100, 246001 (2008).

[10] M. Thiébaud, Z. Shen, J. Harting, and C. Misbah, Phys. Rev. Lett. 112, 238304 (2014).

[11] M. Atif, P. K. Kolluru, C. Thantanapally, and S. Ansumali, Phys. Rev. Lett. 119, 240602 (2017).

[12] C. K. Aidun and J. R. Clausen, Annu. Rev. Fluid Mech. 42, 439 (2010).
[13] T. Krüger, H. Kusumaatmaja, A. Kuzmin, O. Shardt, G. Silva, and E. M. Viggen, The Lattice Boltzmann Method (Springer, Berlin, 2017).

[14] S. Succi, The Lattice Boltzmann Equation (Oxford University Press, Oxford, 2017).

[15] Y.-H. Qian and S. A. Orszag, Europhys. Lett. 21, 255 (1993).

[16] F. J. Alexander, S. Chen, and J. D. Sterling, Phys. Rev. E 47, R2249 (1993).

[17] Z. Guo, C. Zheng, B. Shi, and T. S. Zhao, Phys. Rev. E 75, 036704 (2007).

[18] X. He, S. Chen, and G. D. Doolen, J. Comput. Phys. 146, 282 (1998).

[19] G. R. McNamara, A. L. Garcia, and B. J. Alder, J. Stat. Phys. 81, 395 (1995).

[20] X. Shan and X. He, Phys. Rev. Lett. 80, 65 (1998).

[21] S. Chapman and T. G. Cowling, The Mathematical Theory of Non-Uniform Gases (Cambridge University Press, Cambridge, 1961).

[22] S. S. Chikatamarla and I. V. Karlin, Phys. Rev. Lett. 97, 190601 (2006).

[23] A. Krämer, K. Küllmer, D. Reith, W. Joppich, and H. Foysi, Phys. Rev. E 95, 023305 (2017).

[24] G. Di Ilio, B. Dorschner, G. Bella, S. Succi, and I. V. Karlin, J. Fluid Mech. 849, 35 (2018).

[25] A. Krämer, Lattice-Boltzmann-Methoden zur Simulation inkompressibler Wirbelströmungen, Ph.D. thesis, Universität Siegen, 2017.

[26] See Supplemental Material at http://link.aps.org/ supplemental/10.1103/PhysRevLett.121.130602 for implementation details and further benchmarks, which includes Refs. [27-29].

[27] X. B. Nie, X. W. Shan, and H. D. Chen, Europhys. Lett. 81, 34005 (2008).

[28] N. Prasianakis, S. Chikatamarla, I. Karlin, S. Ansumali, and K. Boulouchos, Math. Comput. Simul. 72, 179 (2006).

[29] F. Bösch, S. S. Chikatamarla, and I. Karlin, ESAIM: Proc. Surv. 52, 1 (2015).

[30] Y.-H. Qian and Y. Zhou, Europhys. Lett. 42, 359 (1998).

[31] G. Házi and P. Kávrán, J. Phys. A 39, 3127 (2006).

[32] S. Ansumali and I. V. Karlin, Phys. Rev. Lett. 95, 260605 (2005).

[33] N. I. Prasianakis, I. V. Karlin, J. Mantzaras, and K. B. Boulouchos, Phys. Rev. E 79, 066702 (2009).

[34] N. Frapolli, S. S. Chikatamarla, and I. V. Karlin, Phys. Rev. Lett. 117, 010604 (2016).

[35] X. Shan, J. Comput. Sci. 17, 475 (2016).

[36] C. Coreixas, G. Wissocq, G. Puigt, J.-F. Boussuge, and P. Sagaut, Phys. Rev. E 96, 033306 (2017).

[37] K. K. Mattila, P. C. Philippi, and L. A. Hegele Jr., Phys. Fluids 29, 046103 (2017).

[38] G. I. Taylor, Aero. Res. Commun. 598, 73 (1918).

[39] O. Inoue and Y. Hattori, J. Fluid Mech. 380, 81 (1999).

[40] N. Frapolli, S. S. Chikatamarla, and I. V. Karlin, Phys. Rev. E 93, 063302 (2016). 